\title{
Uptake of Terminalia chebula Tannin Components by Hide: Analysis Using tlc-uv Densitometry
}

S. Ratnayake and E. R. JANSZ

Natural Products Section, Ceylon Institute of Scientific and Industrial Research, P. O. Box 787, Colombo, Sri Lanka

AND

K. T. D. DE Srlva

Chemistry Department, University of Sri Jayewardenepura, Nugegoda, Sri Lanka

(Date of receipt: 29 July 1982)

(Date of acceptance: 24 May 1984)

\begin{abstract}
A method of separation and quantitative analysis of Terminalia chebula tannins by tlc-uv densitometry is described. The method is applicable over a range of 0.5 to $2.5 \mu \mathrm{g} / \mathrm{tIc}$ spot and has coefficient of variation of $4-6 \%$ for the individual tannins. Using the method on individual tannin components, mixtures of components and myrobalan extracts it has been found that at a level of $0.5 \%$ concentration, chebulinic acid and chebulagic acid are taken up rapidly by hide, their rate of uptake being faster than the products of decomposition (by heat in aqueous solution) of chebulinic acid. The latter indicates an advantage in extracting myrobalan tannins under mild conditions. The study also showed that the optimum $\mathrm{pH}$ for uptake of chebulinic acid by hide is 4.1 indicating that the ionisation of the carboxylic acid group may play a rote in tanning by this compound.
\end{abstract}

\section{Introduction}

Although use of myrobalans, the tannin extract of Terminalia chebula, has been well established in the leather tanning industry ${ }^{5,6}$ and its chemistry also well known for sometime ${ }^{4,10}$ there appears to be little detailed study on the relative tanning value of its tannin components. In fact the studies by Barat ${ }^{1,2}$ as far back as 1953, is one of the few studies of the type reported. In this study, we report an analytical technique for the assay the major tannin components of Terminalia chebula ${ }^{8}$ and its application to situations concerning the tanning of hide using myrobalan extracts. The rate of uptake of tannin components by hide has been specially considered here.

\section{Experimental}

\subsection{Separation of tamin components}

Chebulinic acid was separated from myrobalans by the method of Barat, ${ }^{1}$ while 3,6 digalloylglucose was prepared from chebulinic acid by the method of Freudenberg and Fick. 
Chebulagic acid was isolated from the mother liquor after chebulinic acid precipitation, by the following procedure. The mother liquor $(200 \mathrm{ml})$ was adjusted to $\mathrm{pH} 6.7$ and extracted with ethylacetate $(50 \mathrm{ml} \times 3)$. The aqueous portion was adjusted to $\mathrm{pH} 4.5$ and extracted similarly with ethyl acetate. The $\mathrm{pH}$ was then adjusted to $\mathrm{pH} 2$ and extracted with ethylacetate $(50 \mathrm{ml} \times 3)$. Chebulagic acid was found in the most purified state in the aqueous mother liquor after the final extraction. This was purified by the method of Reddy et al. ${ }^{9}$

\subsection{Experiments with hide}

In order to apply the assay a laboratory model of leather tannin was designed. In most experiments the major tanning components of the extract was maintained at a low level of concentration $(0.5 \%)$ and $10 \%$ acetone added in order to prevent precipitation. Unless otherwise specified, $\mathrm{pH}$ was adjusted to 4.6 .

Into the tannin containing solutions $(100 \mathrm{ml})$ was dipped a small piece ( $5 \mathrm{~g}$ fresh wt., $65-70 \%$ moisture) of chromed hide. Care was taken to keep weight, area and shape of the hide constant. The solution contained in a closed vessel was stirred for $24-48 \mathrm{~h}$ at room temperature $\left(28-30^{\circ} \mathrm{C}\right)$ using a magnetic stirre aliquots withdrawn at various time intervals. The aliquots were diluted with ethanol to the range of 0.5 to $2.5 \mu \mathrm{g} /$ tannin component concerned and spotted on a tlc plate.

\subsection{TLC-UV densitometry}

Separations were carried out using $500 \mu$ silica gel $G_{60}$ tlc plates developed in ethyl formate: formic acid: toluene $(2: 1: 1)$. The tannins in ethanolic extract were spotted on the tlc plates so that the content of each component tannin per spot was no more than $2.5 \mu \mathrm{g}$. Using this technique, the myrobalans tannin clearly separated into 2 major and 6 minor spots. In order of increasing Rf value the spots were : chebulic acid, 1, 3, 6-trigalloylglucose, chebulagic acid (major) chebulinic acid (major) 3, 6-digalloylglucose, unknown glucogallin and gallic acid.

The plates were allowed to dry in the dark for 5-10 min and then dried further with an even stream of hot air for 3-5 min. The tannins then autooxidise to produce grey spots. Uv-densitometry was carried out on the tlc plates immediately after colour development.

The instrument used for tlc-uv densitometry was a Camag automatic scanning, variable wavelength densitometer (model 76500) equipped into a strip chart recorder (Camag, $w+w 1107$ ) at $625 \mathrm{~mm}$. (Band with $30 \mathrm{~nm}$, slit $6 \mathrm{~mm}$ ). Standard curves were plotted for chebulinic acid, chebulagic acid, digalloylglucose and gallic acid. The curves were linear in the range of 0.5 to $2.5 \mu \mathrm{g} / \mathrm{spot}$ and the coefficient of variation was $4-6 \%$. As there were small variations from plate to plate 
calculations were made by comparing spots on the same plate and expressing results as a percentage of the control by running standards on the same plate and rejecting all readings out of the normal range of linearity. Samples were run in duplicate and values recorded are a mean of duplicate values which agreed to within $5 \%$ crror.

\section{Results}

\subsection{Sri Lankan myrobalans-main tannin components}

The main components were chebulinic acid (approximately $14 \%$ ) and chebulagic acid (approximately $7 \%$ ); all other components were less than $2 \%$. All results are expressed on dry weight of the fruit. Drum drying of extracts results in decomposition of $15-20 \%$ of the chebulinic acid and the appearance of digalloylglucose.

\subsection{Effect of heat on aqueous chebulinic acid solutions}

Chebulinic acid solution when heated at temperatures of $70^{\circ} \mathrm{C}$ and $80^{\circ} \mathrm{C}$ for 8 hours does not produce 3,6-digalloylglucose but another compound suspected to be $1,3,6$-trigalloylglucose as it is derived from chebulinic acid and decomposes to give equimolar amounts of digalloylglucose and gallic acid on decomposition. At temperatures of $90^{\circ} \mathrm{C}$ and $100^{\circ} \mathrm{C} \mathrm{3,6-digalloylglucose} \mathrm{is} \mathrm{produced} \mathrm{(Table} \mathrm{1).}$

TABLE 1. Effect of heating a dilute chebulinic acid solution

\begin{tabular}{|c|c|c|c|c|c|c|c|c|c|c|c|c|}
\hline Temperature $\left({ }^{\circ} \mathrm{C}\right)$ & \multicolumn{3}{|c|}{70} & \multicolumn{3}{|c|}{80} & \multicolumn{3}{|c|}{90} & \multicolumn{3}{|c|}{100} \\
\hline Time (hours) & 0.5 & 2 & 8 & 0.5 & 2 & 8 & 0.5 & 2 & 8 & 0.5 & 2 & 8 \\
\hline Digalloylglucose $\left(\mathrm{mm}^{2}\right)$ & 0 & 0 & 0 & 0 & 0 & 12 & 0 & 0 & 78 & 0 & 28 & 92 \\
\hline Trigalloylglucose $\left(\mathrm{mm}^{2}\right)$ & 0 & 27 & 44 & 18 & 49 & 82 & 34 & 57 & 54 & 37 & 56 & 34 \\
\hline
\end{tabular}

Chebulinic acid $(0.1 \%)$ in water was maintained at the above temperatures for the given times and aliquots analysed by tlc-uv densitometry.

Concentration is referred to in terms of peak area $\left(\mathrm{mm}^{2}\right)$. For digaloylglucose and trigalloyl. glucose $100 \mathrm{~mm}^{2}$ is approximately $1 \mu \mathrm{g}$. For the same aliquot, original chebulinic acid peak area$158 \mathrm{~mm}^{2}$. Residual chebulinic acid (after 8 hours) was $45,10,0$ and $0 \%$ at $70,80,90$ and $100^{\circ} \mathrm{C}$ respectively.

\subsection{Uptake of individual tannins by hide}

During preliminary experiments uptake of tannin components was monitored by measuring optical density of the solution at $277 \mathrm{~nm}$ after dilution. Loss of tannins from the solution was assumed as indicative of uptake of tannins by hide, as control experiments (without hide) resulted in no significant losses of tannins on stirring even after 48 hours and further that hide released $277 \mathrm{~nm}$ absorbing substances to 
the medium to a very small extent (less than $2 \%$ total absorbance) upto 24 hours. Results (Figure 1) shows that chebulagic acid was taken up by hide more rapidly than either chebulinic acid or digalloylglucose.

Studies also showed that increases in concentration of chebulinic acid to $2.5 \%$ resulted in marked increases in the rate of uptake. Notwithstanding the controls used it was felt that anomalies could be experienced; for example, as a result of reaction between substances released by hide and the component tannin thus producing $277 \mathrm{~nm}$ absorbing material. This was verified when chebulinic acid uptake was followed using tlc-uv densitometry (Figure 1). Similar results were obtained but uptake was greater by this method for readings taken beyond 8 hours, confirming interference by non-chebulinic $277 \mathrm{~nm}$ absorbing components in the direct uv method. Thus the advantages of using the tlc-uv densitometric technique where such impurities are separated by tlc and the tannin concerned quantified independently is emphasised.

\subsection{Effect of $\mathrm{pH}$}

Results (Figure 2) showed that a pH optimum of 4.1 was shown tor the uptake of chebulinic acid.

\subsection{Dual components}

Studies using mixtures of chebulinic acid and digalloylglucose confirmed that the former was bound by hide to a much greater extent (Figure 3). Studies using a mixture of chebulinic acid and its decomposition product (at $70^{\circ} \mathrm{C}$ ), suspected to be trigalloylglucose, showed that chebulinic acid exhibits a far higher rate of uptake (Figure 3).

\subsection{Myrobalan extracts}

The use of these extracts have the advantage of the presence of non-tannins and minor components of tannin value and therefore results are of added significance. Study of uptake of tannin components by hide by a myrobalan extract clearly showed that uptake of chebulinic acid was most significant (Figure 4). As this may be merely an effect of higher concentration, the same experiment was conducted: with the mother liquor after chebulinic acid extraction which had approximately the same molar concentration of the two components. Results again showed that the rate of chebulinic acid uptake was much higher than that of chebulagic acid (Figure 4).

\section{Discussion}

The fact that chebulinic acid appears to be taken up by hide faster than chebulagic acid in mixed extracts, while the opposite is true for the component tannin in the pure form is indicative of the complex nature of tannin uptake by hide. However, 


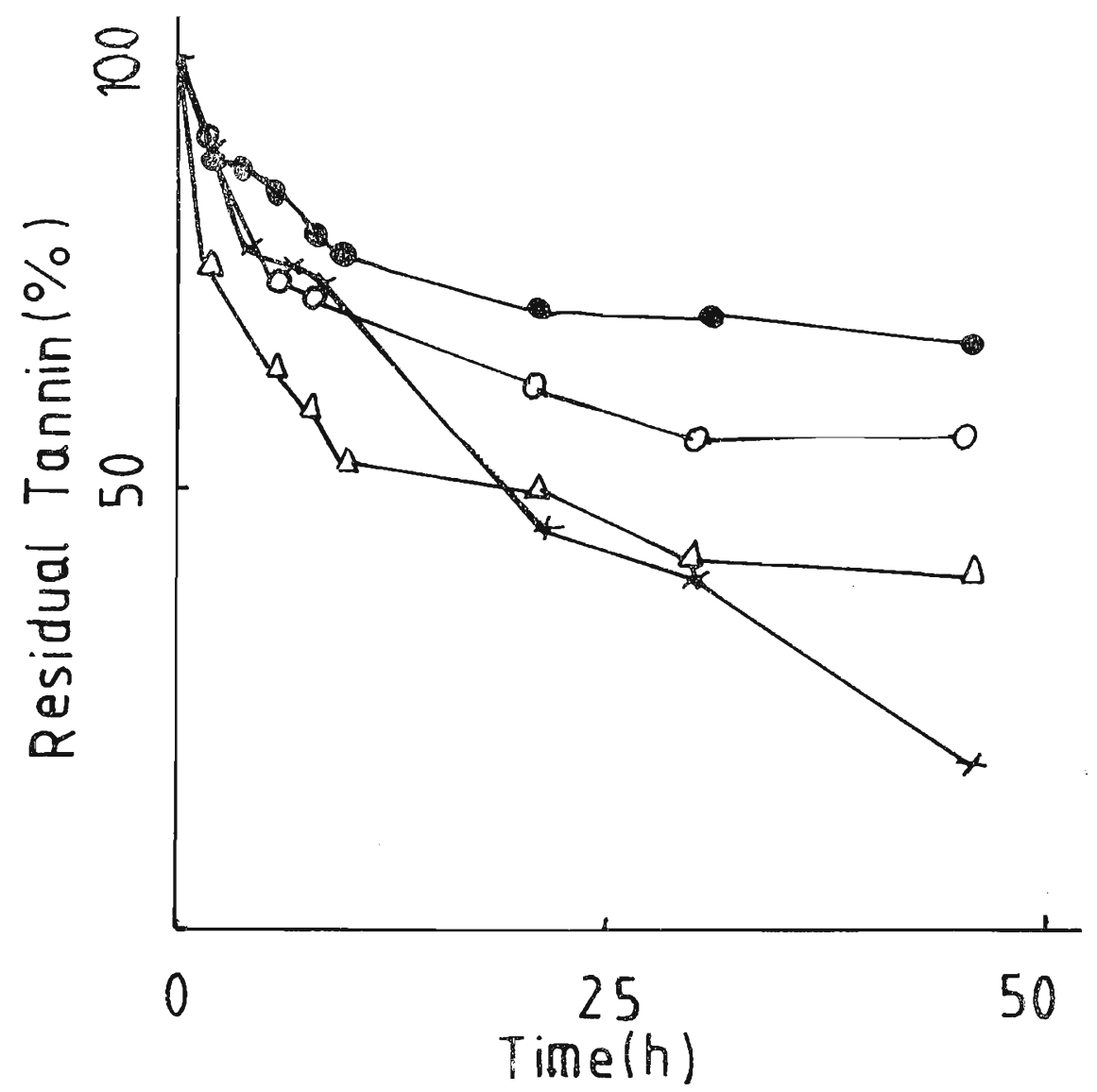

Figure 1 - Uptake of individual tannin by hide

$x-x$, Chebulinic acid by tlc-uv densitometry

$\mathrm{O}-\mathrm{O}$, Chebulinic acid by direct UV absorbence

A Chebulagic acid by direct UV adsorbance

- Digalloylglucose by direct UV absorbance

Uptake was calculated by measuring residual tannin in medium by the method given above. $100 \%$ refers to $5 \mathrm{mg} \mathrm{cc-}^{-2}$ tannin concentration. Other details as in experimental. 


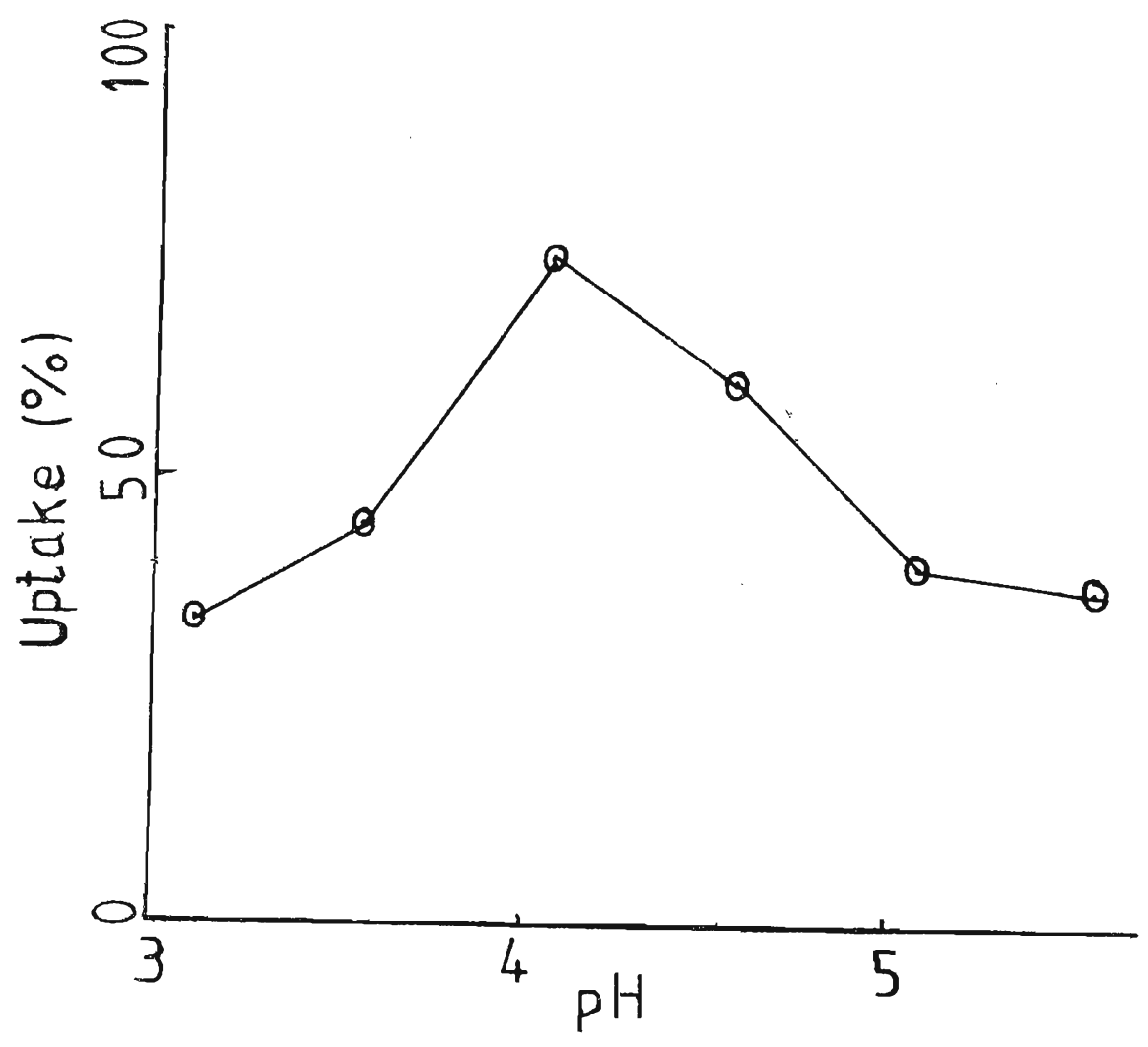

Figure 2 - Effect of $\mathrm{PH}$ on chebulinic acid uptake by hide.

$\mathrm{P}^{\mathrm{H}}$ was maintained by use of $2 \mathrm{~m}$ Acetate buffer. Original chebulinic acid concentration $=5 \mathrm{mg} \mathrm{cc}^{-1}$. Uptake was calculated by measuring residual tannin in medium by tlc-uv densitometry over a time course of 8 hours. Uptake at 4 hours is plotted versus pr. 


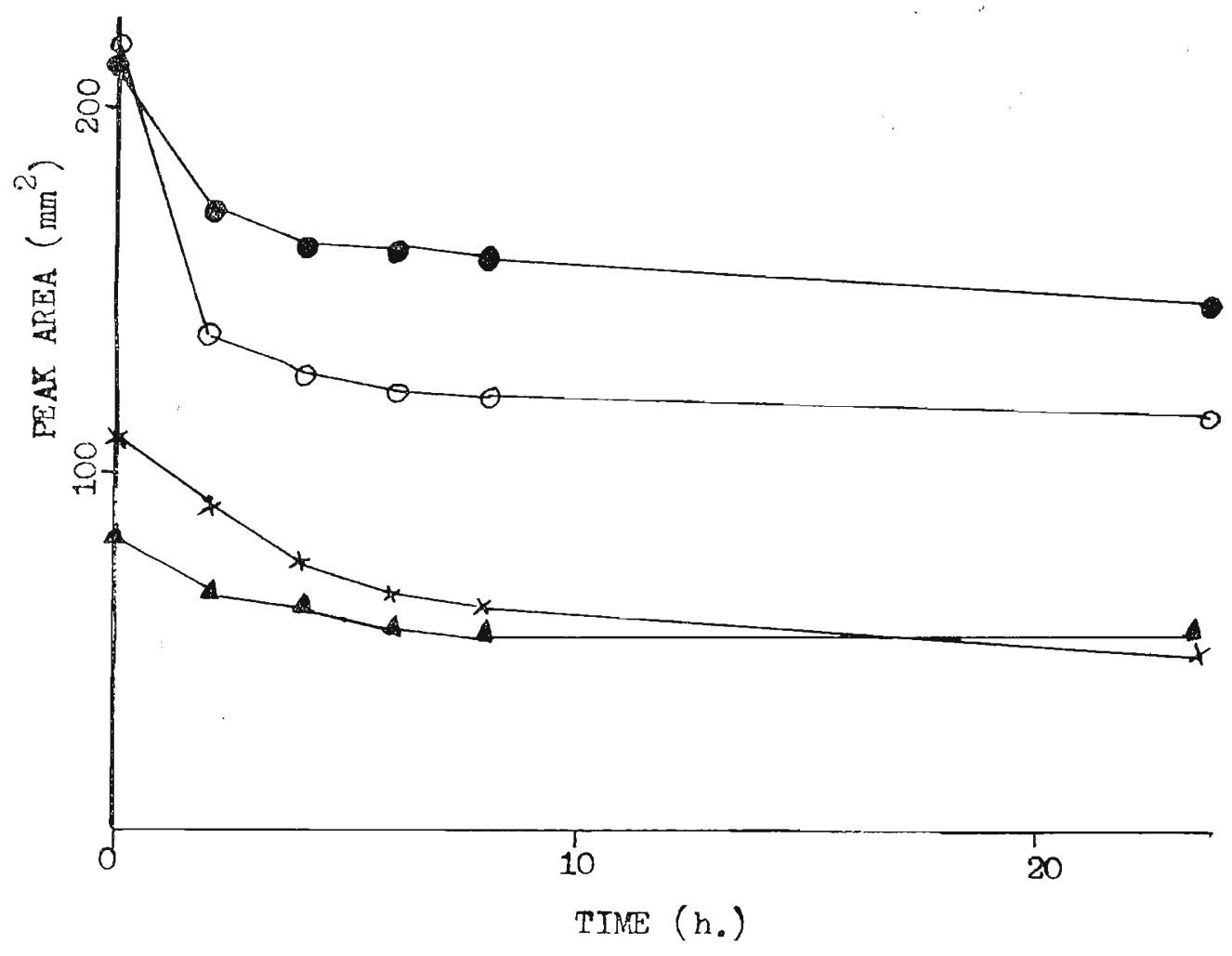

Figure 3 -.. Uptake of selected myrobalan tamnin components

Expt A Concentration is expressed in $\mathrm{mm}^{2}$ - A peak area of $100 \mathrm{~mm}^{2}$ represents approxima. tely $1.5 / \mu \mathrm{g}$ chebulinic acid and $1 / \mathrm{\mu g}$ digalloylglucose in an aliquot. Initial concentration of chebulinic acid and digalloylglucose was approximately $2.0 \mathrm{~mm}$ and $2.5 \mathrm{~mm}$ respectively.

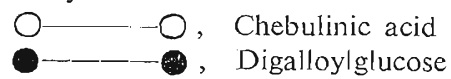

Expt B A peak area of $50 \mathrm{~mm}$ is approximately $1.5 / \mathrm{\mu g}$ for chebulinic acid and trigalloylglucose respectively. Initial concentration of the two compounds were $5 \mathrm{~mm}$ and $4 \mathrm{~mm}$ respectively

$\mathbf{A}$, Chebulinic acid;

$\times-\times$, Trigalloylglucose. 


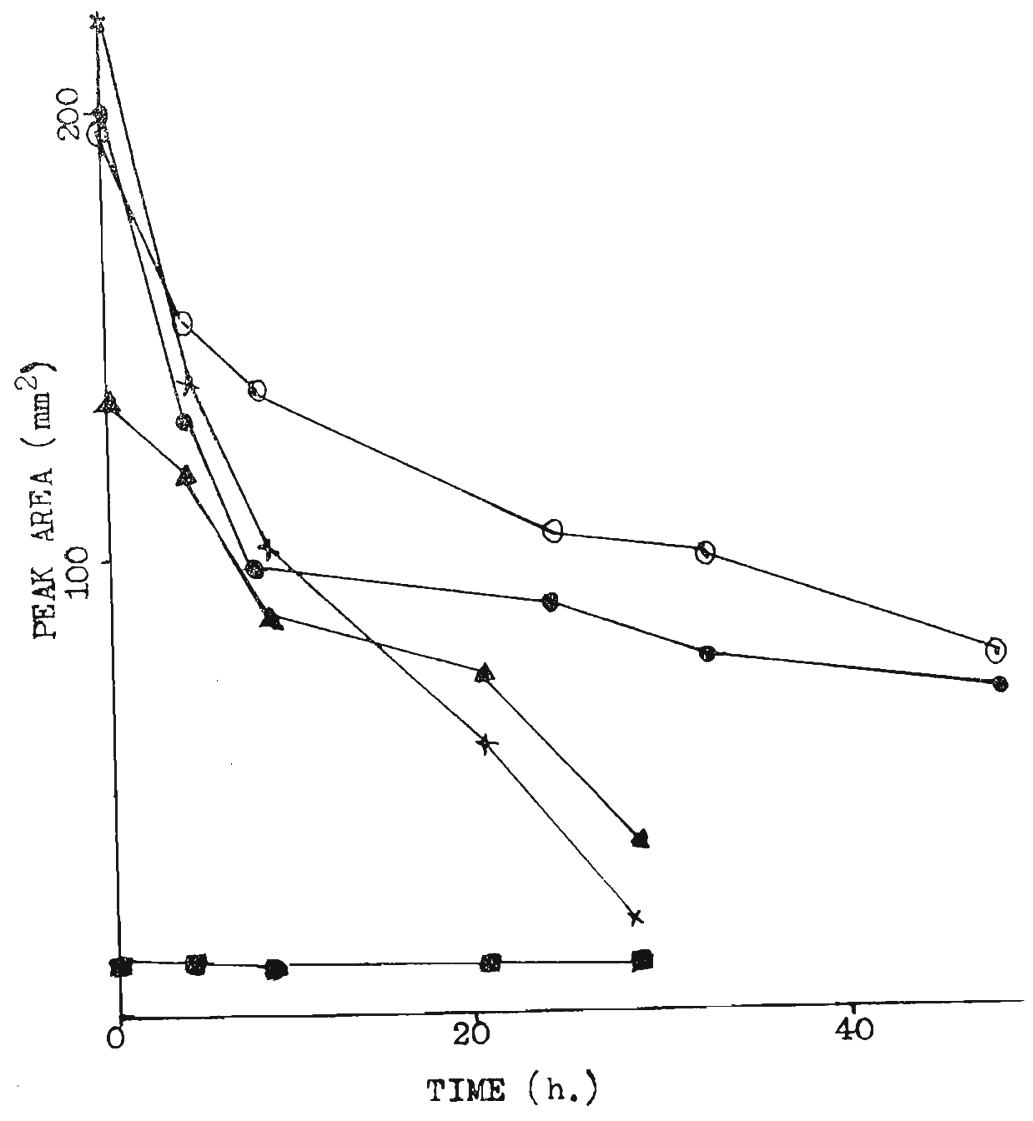

Figure 4 - Uptake of selected tannin/ from myrobalan extracts

A A myrobalan extract (10 $\mathrm{mg} \mathrm{cc}^{-1}$ tannin) was agitated with hide and an aliquot assayed.

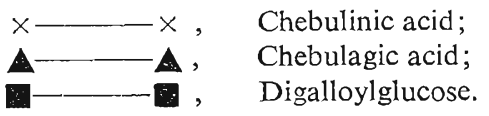

B The mother liquor obtained after precipitation of chebulinic acid by the method of Barat ${ }^{1}$ was used. Tannin concentration of the mother liquor (as determined by the hide powder method) was diluted to $10 \mathrm{mg} \mathrm{cc}^{-1}$.

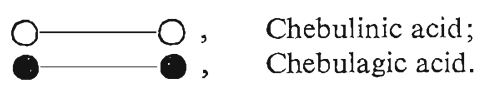

$100 \mathrm{~mm}^{2}$ is equivalent to approximately $1.5,1.5$, and $1 \mu \mathrm{g}$ chebulinic acid, chebulagic acid and digalloylglucose respectively. 
it must be pointed out that the experimental designs of experiments involving pure components on one hand and extracts on the other have one major difference and that is the presence of acetone in the former (to attain solubility). This factor could introduce differences that influence the rate of uptake, since it is highly probable that myrobalan extracts are not true solutions but polydisperse agglomerates. Another factor that could influence rates of uptake of tannin components is the presence of non-tannins and minor tannins in extracts but not in the case of the pure components.

The uptake (by hide) of chebulinic acid decomposition products (di - and trigalloylglucose) is lower than that of chebulinic acid. Taken together with earlier findings 7,9 namely that the two other decomposition products-gallic acid and chebulic acid have no tanning value, this leads us to the conclusion that any operation that leads to decomposition of chebulinic acid will result in reduced tanning value of the myrobalan extract. This conclusion is consistent with previous findings? that tannin content (as determined by the hide powder method) is highest in extracts made at $80^{\circ} \mathrm{C}$ for $15 \mathrm{~min}$; an increase in either temperature or time resulting in lowering of tannin content in solution.

In this study the $\mathrm{pH}$ optimum obtained for maximum chebulinic acid uptake strongly suggests that the ionisation of the carboxylic acid group plays some role in tanning by this compound.

\section{Acknowledgements}

The authors thank the Director, CISIR, for facilities provided, the Ministry of Industries and Scientific Affairs, Sri Lanka for a special grant for this study. The authors also thank Misses Dinapali de Zoysa and Ramani Perera for secretarial assistance. This work is a part of the CISIR research programme and the MSc thesis, Sri Jayawardenapura University, of S. Ratnayake.

\section{References}

1. Barat, S. K. (1953). Ind. Chem. Soc. (Ind. and News Ed.) 16:9.

2. Barat, S. K. (1953). Ind. Chem. Soc. (Ind. and News Ed) 16: 10.

3. Frendenberg, K. \& Fick, B, (1920). Ber, 5: 3 1728-36 (CA 1921 15, 846) and as quoted by DE SILVA L. B., (1953) PhD thesis University of Sheffield, "Some constituents of myrobalans tannins".)

4. Haworth, R. D. (1963). Advancement of Sci. 81: 396.

5. Kanial, G. \& Nayudamma, Y. (1966). J. Sci. Ind. Res. 25: 400.

6. Kedlaya, K. J. (1970). Leather Sci. 18: 396.

7. Ratnayake, S. \& Jansz, E. R. (1981). Proceedings of the 10th Annual Sessions, Institute of Chemistry (Sri Lanka).

8. Ratnayake, S., Jansz, E. R. \& de Silva, K. T. D. (1982). Proceedings of the 1lth Annual Sessions of the Institute of Chemistry (Sri Lanka).

9. Reddy, K. K., Rajadurai, S. \& Nayudamma, Y. (1965). Indian J. Chem. 3: 129.

10. WhIte, T., (1956). In "The Chemistry of tannins - A Symposium" 12;13 Cambridge University, Croydon. 